\title{
Survey of Mechatronic Techniques in Modern Machine Design
}

\author{
Devdas Shetty, ${ }^{1}$ Lou Manzione, ${ }^{2}$ and Ahad $\mathrm{Ali}^{3}$ \\ ${ }^{1}$ School of Engineering and Applied Sciences, University of the District of Columbia, Washington, DC 20008, USA \\ ${ }^{2}$ Department of Mechanical Engineering, University of Hartford, West Hartford, CT 06117, USA \\ ${ }^{3}$ A. Leon Linton Department of Mechanical Engineering, Lawrence Technological University, Southfield, MI 48188, USA \\ Correspondence should be addressed to Ahad Ali, sali@ltu.edu
}

Received 26 July 2012; Revised 25 October 2012; Accepted 8 November 2012

Academic Editor: Yangmin Li

Copyright (๑) 2012 Devdas Shetty et al. This is an open access article distributed under the Creative Commons Attribution License, which permits unrestricted use, distribution, and reproduction in any medium, provided the original work is properly cited.

Increasing demands on the productivity of complex systems, such as manufacturing machines and their steadily growing technological importance will require the application of new methods in the product development process. A smart machine can make decisions about the process in real-time with plenty of adaptive controls. This paper shows the simulation based mechatronic model of a complex system with a better understanding of the dynamic behavior and interactions of the components. This offers improved possibilities of evaluating and optimizing the dynamic motion performance of the entire automated system in the early stages of the design process. Another effect is the growing influence of interactions between machine components on achievable machine dynamics and precision and quality of components. The examples cited in this paper, demonstrate the distinguishing feature of mechatronic systems through intensive integration. The case studies also show that it will no longer be sufficient to focus solely on the optimization of subsystems. Instead it will be necessary to strive for optimization of the complete system. The interactions between machine components, the influence of the control system and the machining process will have to be considered during the design process and the coordination of feed drives and frame structure components.

\section{Simulation of Complex Structures}

Mechatronics is a methodology used for the optimal design of electromechanical products. The term was coined nearly 40 years ago, in 1969, when the engineer Tetsuro Mori combined the words "mechanical" and "electronic" to describe the electronic control systems that Yaskawa Electric Corporation was building for the mechanical factory equipment. Mechatronics is a design philosophy, which is an integrating approach to an engineering design as shown in Figure 1. The primary factor in mechatronics is the involvement of these areas throughout the design process. Through a mechanism of simulating interdisciplinary ideas and techniques, mechatronics provides ideal conditions to raise the synergy, thereby providing a catalytic effect for the new solutions to technically complex situations. An important characteristic of the mechatronic devices and systems is their built-in intelligence that results through a combination of precision mechanical and electrical engineering and realtime programming integrated to the design process. The synergy can be generated by the right combination of parameters; that is, the final product can be better than just the sum of its parts. Mechatronic products exhibit performance characteristics that were previously difficult to be achieved without the synergistic combination. Recently, some mechatronic applications are presented on micromotion and helicopter and robotic arm in different research articles [1-5].

A typical mechatronic design process [6] is shown in Figure 2. Starting with steps 5 through 9 , software tools are available to aid the designer in creating and debugging the mathematical system models. Some tools that are particularly useful allow the designer to represent the system by creating a system block diagram from simple building blocks such as integrators, gain stages, summing junctions, and nonlinear switches. Some examples of these tools are LabVIEW, Simulink/Matlab, Matrix-x, ACSL, SimPACK, Hypersignal, and VisSim. These graphical simulation tools run on generic platforms such as desktop PC compatible Windows operating systems. With any of these tools, 


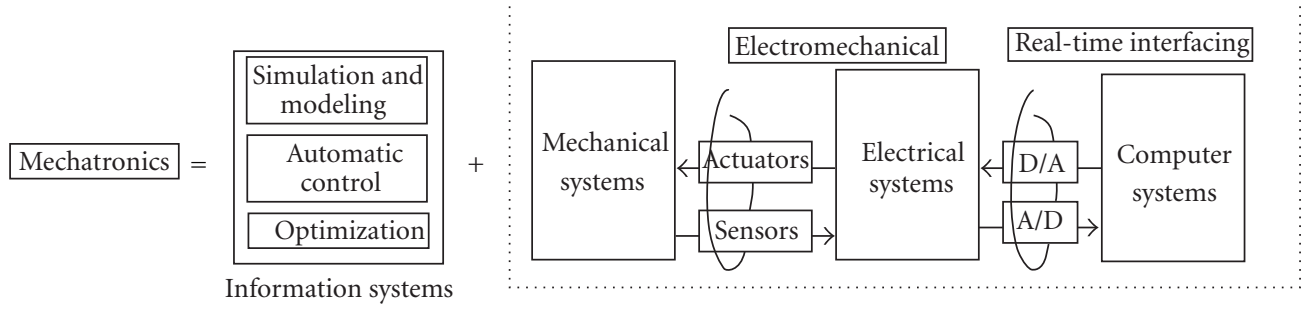

Figure 1: Mechatronic Process.

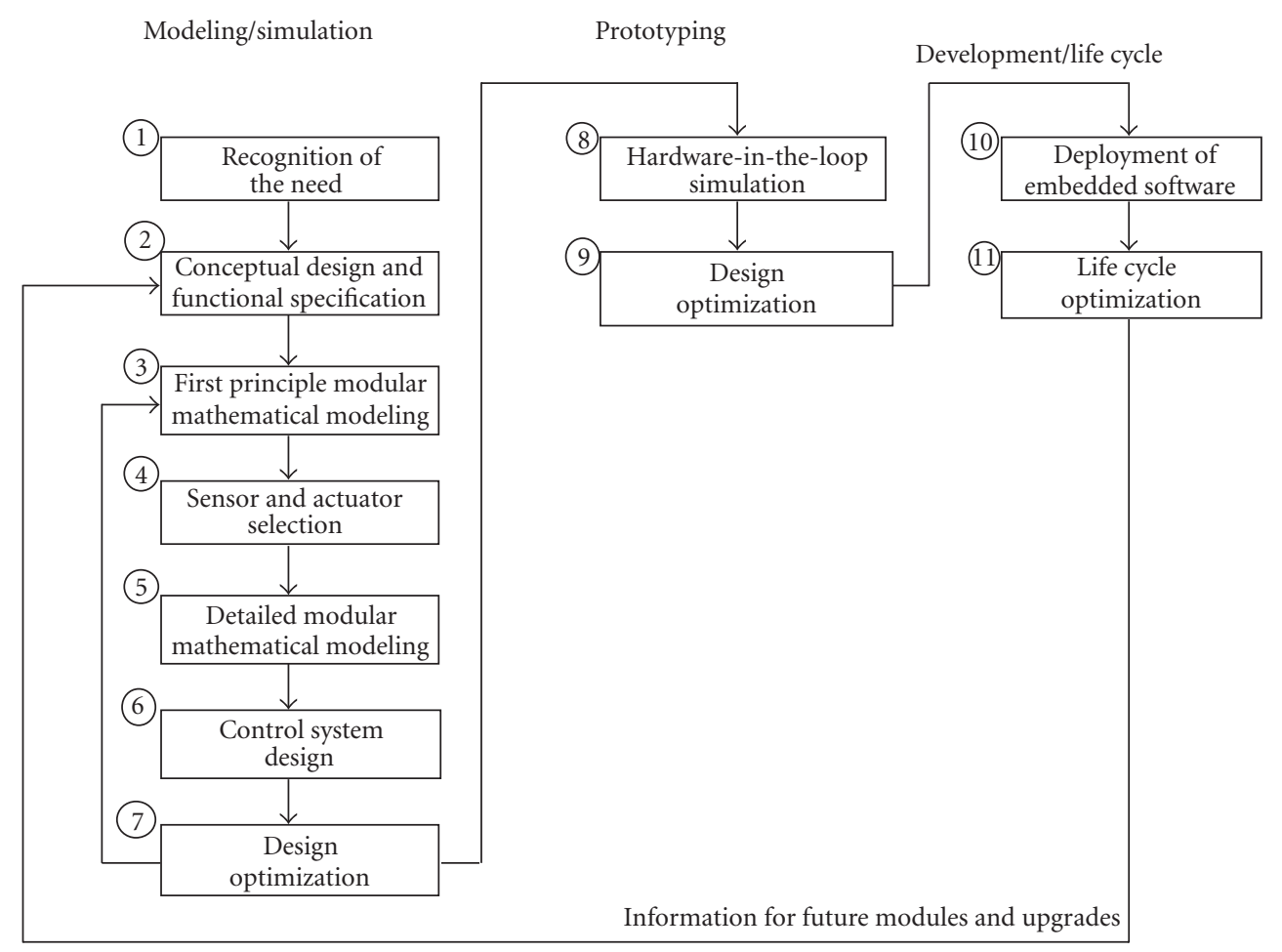

Figure 2: Mechatronic design [6].

the designer can create a plant model and then validate it against real-world measurements (step 5). Once the plant model has been validated, the designer can then design the control system and optimize it until the correct response is achieved (steps 6 and 7). In some cases, completely accurate plant models cannot be made and certain assumptions must be made about that plant model that cannot be validated. In these cases, it is advantageous to be able to test the control system within the plant environment (step 8). This is sometimes referred to as hardware-in-the-loop simulation since some of the actual hardware (mechanical and electrical parts) is used in the system control loop (acting as the plant that is to be controlled). The hardware-in-the-loop simulation testing provides the designer with reassurance that any assumptions made on the plant model were correct. If any assumptions were incorrect, however, the designer has the opportunity to optimize the design (step 9) before committing.

\section{Design of Smart Machine Tools Using Mechatronics}

Researchers used mechatronic techniques for different applications. One of the applications is for smart machine tool design [7]. It explains the application of integrated CAx tools for setting up a virtual prototype that will permit evaluation and optimization of the entire machine tool's motion dynamics in early phases of the development process. Due to the development of machine design and drive technology, modern numerically controlled machine tools can be described to an increasing extent as characteristic examples of complex mechatronic systems [8]. A distinguishing feature of mechatronic systems is the achievement of system functionality through intensive integration of electronical and information subfunctions on a mechanical carrier [9].

In another research, tool path interpolation of the $\mathrm{NC}$ motion control is of great importance for the obtainable 


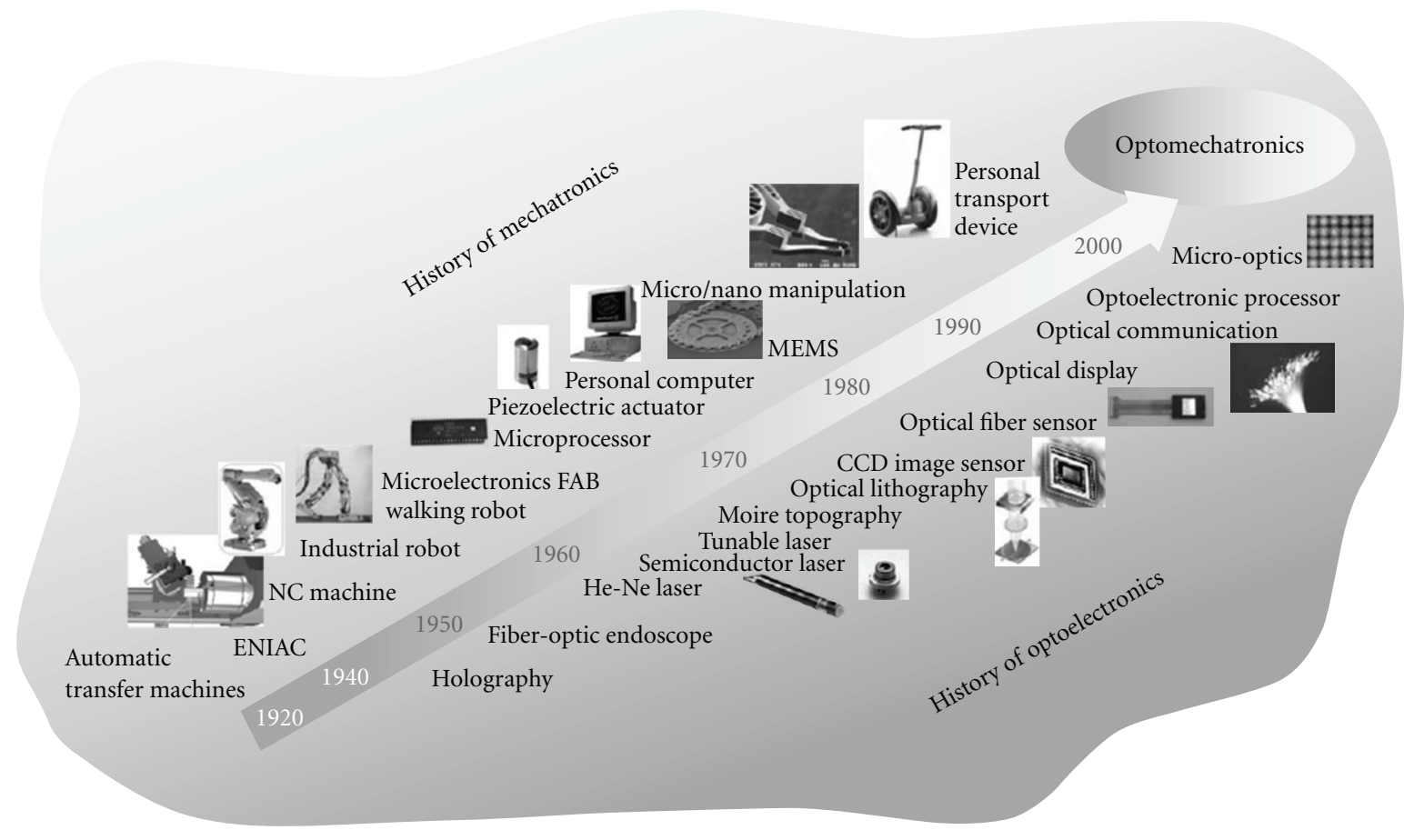

FIGURE 3: History of optomechatronics [20].

motion dynamics and the resulting contouring error, especially for highly dynamic machine movements. The contouring error consists of the tracking error of the feed axes and of the deflections of the TCP caused by the physical effects of the flexible machine structure. In order to reduce the contouring error, modern NC controllers make use of two major technological approaches [10].

Optical technology has been incorporated into mechatronic systems at an accelerated rate, and as a result, a great number of machines/systems with smart optical components have been presented which introduces the fundamental concept, definition, and characteristics of the technology by analyzing the characteristics of a variety of practical optomechatronic systems [11]. Optical elements have been increasingly incorporated at an accelerated rate into mechatronic systems, and vice versa [12-19].

Optomechatronics has its roots in technological developments of mechatronics and optoelectronics. Figure 3 shows the chronology of those developments [20]. In the 1960s, the electronic revolution came with the integration of transistor and other semiconductor devices into monolithic circuits, and, in 1971, the semiconductor fabrication technology brought about a tremendous impact on a broad spectrum of technological fields. In the 1980s, the semiconductor technology also created microelectromechanical systems (MEMS), and this brought about a new dimension of machines/systems, microsizing their dimension.

The major functions and roles of mechatronic elements in optomechatronic systems can be categorized into the following five technological domains [21]: sensing, actuation, information feedback, motion/state control, and embedded intelligence with microprocessor.
In the last few years the Virtual Design of machine tools has been extensively studied in several manufacturing automation and production engineering laboratories of universities and research institutes. This new technology is mainly studied and applied to machining centers (MCs) for high speed milling (HSM), the manufacturing of complex dies, moulds and aerospace parts currently being a strategic sector of production engineering. Almost a hundred relevant papers $[7,22-25]$ have recently been published on these topics in leading engineering journals and presented at several technical conferences; this confirms the significant interest, both industrial and academic, in virtual design.

For CNC manufacturers and MC users, however, the full exploitation of virtual machine tool technology still requires [22] fundamental developments mainly in the areas of cutting process simulation and full integration of all analysis modules in a user-friendly environment. The integration of the two models in one simulation environment is now possible, making it possible to study the interactions between the dynamics of these active and passive mechanical structures $[22,26]$. The optimization of their performances is a basic prerequisite for ensuring productivity at shop floor level: fast machining times, the required dimensional accuracy, and good surface quality of the workpieces. Gurbuz [27] presented the mechatronic approach for desktop CNC milling machine design.

Siemens introduced mechatronics concept designer with a new integrated machine design solution that develops and markets machine tools and production machines [28]. The mechatronics concept designer represents a paradigm shift for the industry with a new system engineering approach to machine design that captures "voice of the customer" input, 


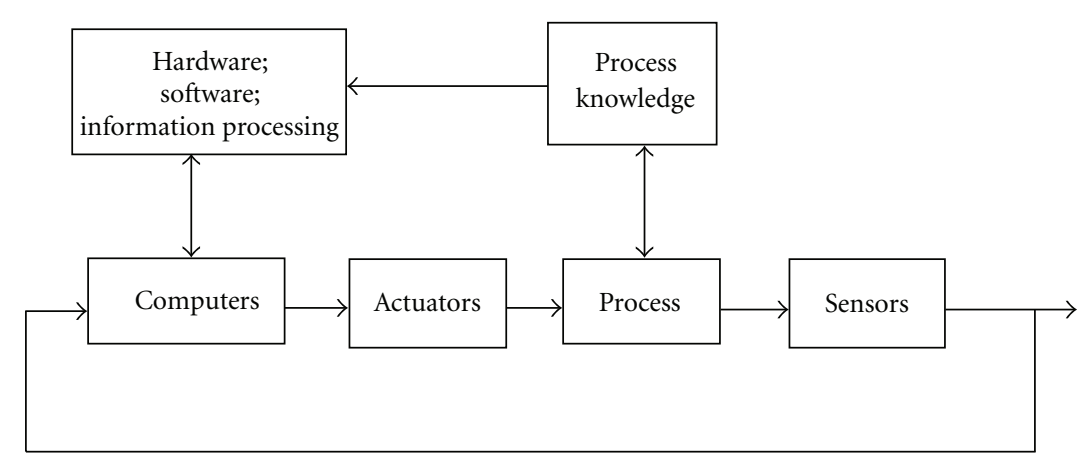

FIgURE 4: Hardware and software integration.

manages early requirements, and facilitates the simultaneous definition and simulation of the complex mechanical, electrical, and automation software found in today's increasingly complex machine tools. With an easy-to-use, interactive simulation capability based on groundbreaking "gaming" technology, the mechatronics concept designer can help significantly reduce development time and improve product quality for the global machine design industry.

\section{Integrated Design Issues in Mechatronics}

The integration within a mechatronic system can be performed through the combination of hardware (components) and software (information processing). Hardware integration results from designing the mechatronic system as an overall system and bringing together the sensors, actuators, and microcomputers into the mechanical system. Software integration is primarily based on advanced control functions. Figure 4 illustrates how the hardware and software integration takes place. It also shows how an additional contribution of the process knowledge and information processing is involved beside the feedback process. A mechatronic product can achieve impressive results if it is effectively integrated with the concurrent engineering management strategy. The benefits that accrue are greater productivity, higher quality, production reliability by the incorporation of intelligent, and self-correcting sensory and feedback system. The basic approach involves accurate computer-based dynamic models from illustrations and other information using the analogy approach. This unique method combines the standard analogy approach to modeling, with block diagrams, the major difference being the ability to incorporate nonlinearities directly into the system without linearization. Control system design methods are available with several design procedures for common control structures including PID, Lag, Lead, Rate Feedback, and pure gain. Signal processing and data interpretation are also handled using the visual programming approach. The hardware-in-the-loop simulation testing provides the designer with reassurance that any assumptions made on the plant model were correct. If any assumptions were incorrect, however, the designer does have the opportunity to optimize the design before committing to the real target hardware platform.
There are two main methods currently used to accomplish the hardware-in-the-loop simulation testing. One utilizes the desktop/workstation graphical user interface (GUI) coupled with standard data acquisition and control (DAC) interface circuit boards. One major drawback that simulation software and other PC based simulation systems suffer from is the inability to work in systems where loop responses need to be fast. Therefore once the control algorithms are designed and debugged, the algorithms must then be reimplemented, retested, and debugged on an embedded platform. The second method for hardware-in-the-loop testing involves crosscompiling the control algorithm to target an embedded realtime processor platform. The embedded processor platform is often a digital signal processor (DSP), with $\mathrm{I} / \mathrm{O}$ that is tailored for embedded system products. Embedded processor platforms are designed for reduced cost and increased speed, and as such they generally do not have video displays nor standard desktop inputs such as full function keyboards and mouse interfaces. Figure 5 shows a typical setup for a DSPbased hardware-in-the-loop testing.

The main elements of mechatronic approach are as follows

(i) Demonstration of a language-neutral approach to code system development. This is done using visual simulation.

(ii) Use of system dynamics and computer simulation in the system development.

(iii) Building of the smart sensor. (Examples are DSPbased velocity probes and accelerometers.)

3.1. Open Architecture with Mechatronic Models: Speed and Complexity [29]. Mechatronics plays the role irrespective of the possibility of single or multiple microcontrollers handling machine tools or an automobile assembly line of multiple robots. Simulating such complex systems allows designers to develop system without finalizing the hardware. The simulation procedure can be as "what if" scenario when the hardware does not exist. There are two critical issues to consider: speed and complexity. Larger systems involve more detailed simulation and specific system requirements. Tradeoffs between simulation speed and the level of accuracy are necessary because of system resources available. 


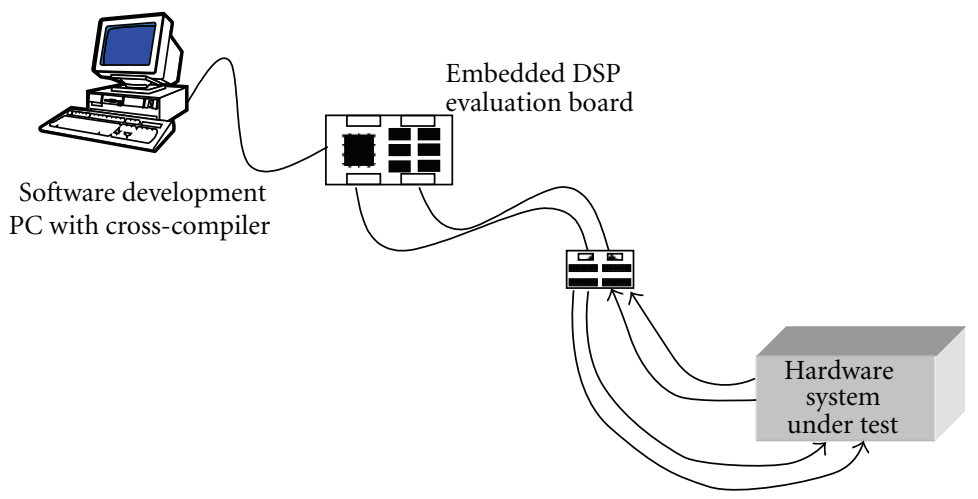

FIGURE 5: Embedded DSP-based "hardware-in-the-loop simulation.”

The simulation becomes faster with faster processors, but the use of multicore systems helps simulation. This is because the systems being simulated are distributed as well. Figure 6 shows an example of Stewart platform which is used in production lines and in many other industrial applications. The Stewart platform is a kind of parallel manipulator using an octahedral assembly of struts. A Stewart platform has six degrees of freedom $(x, y, z$, pitch, roll, and yaw). In this case, there are effectively two models: the simulated physical model and the application model. The physical model accounts for the physics-based simulated environment. The application model interacts with this environment to simulate the real-word application. Simulink and Matlab are used as model-based development tools, so the application is a model. The basic design represented in the physical world by computer-aided design and manufacturing tools (such as CATIA, Autodesk, and SolidWorks) has advanced simulation tools, although they are oriented toward physical construction rather than process control integration [29].

The simulation platform can examine stress under a dynamic loading condition. It also addresses nonlinear analysis like deflection and impact with flexible materials such as foam, rubber, and plastic. In many cases, simulation and analysis of physical entities is useful in a design that does not include a computer-based controller. The contribution by National Instruments facilitates a major integration which facilitates the design engineers to bring in mechanical elements such as gears, cams, and actuators, while the programmers concentrate on the feedback and control algorithms that will handle the motors and actuators in the system. By linking various objects together we enable the models to interact. The provision of rendering permit the visualization of the models is in action. When creating large models, the modeling environment can demand significant amounts of computing power. The creation of large models can be a challenge to computing. At this stage, open architecture hosts can make a significant difference.

3.2. Multicore Speed Simulation [30]. Several CAD and model-based design systems employ interface software that takes advantage of multiple cores. New efforts are under

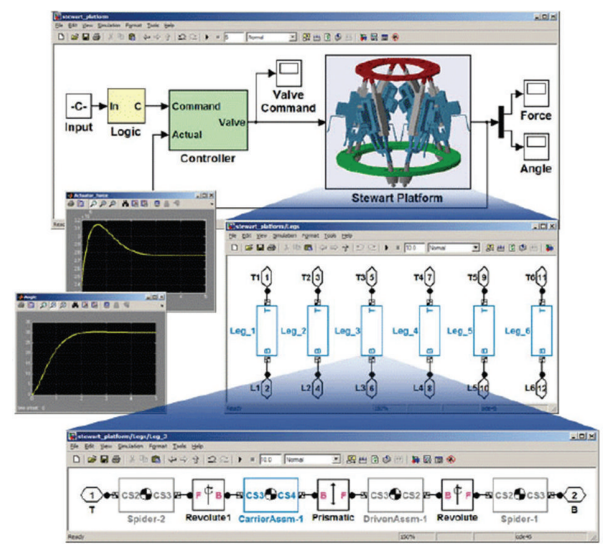

FIGURE 6: Simulink model of a platform [30].

way to develop and link several cores. Exploiting a large number of cores and clustered systems had been a challenge in advanced software architectures. The major challenge is communication between cores. The basic requirement of mechatronic simulation is the time synchronization between various objects in a distributed environment. Simulation in a multiple core environment is again a challenge when the shared memory cannot handle the synchronization. Typically there is an amount of limitation in the physical space. A robotic line-assembly simulation can perform well within its region, but will have a limited capability if it has to interact with other cells. Graphical model-based programming can assist in linking multiple cells (Figure 7).

3.3. Interactive Modeling. The key aspect of the graphical environments is that the visual representation of system partitioning and interaction lends itself to mechatronic applications. They also reduce system complexity from a developer's standpoint, allowing concentration on the application details. For example, the simulation tool such as Simscape is used as a declarative language that defines implicit relationships between components versus the explicit programming specifications for languages like $\mathrm{C}$ and $\mathrm{C}++$ as well as graphical dataflow languages such as LabVIEW. Simscape targets cosimulation where programming and CAD 


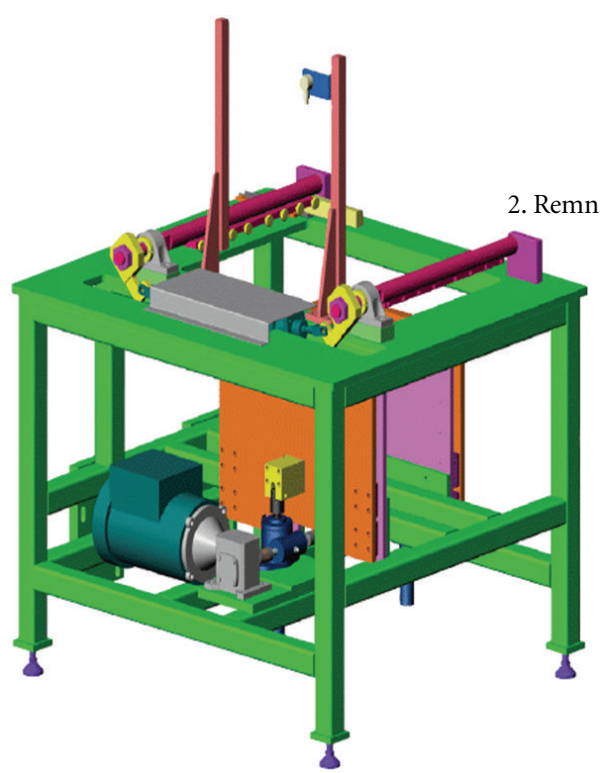

Figure 7: Assembly line design using "Cad” models [30].

intersect. This multidomain tool ties together the electronics, mechanical drive elements, mechanics, and hydraulics tools. For example, Stewart platform simulation discussed earlier can incorporate electrical, hydraulic, mechanical, and signal flow support in addition to software control of the system.

By reducing the amount of expertise required for developing mechatronic applications, developers can spend time and effort on other areas where they do have expertise. Likewise, having a model environment permits a better exchange of ideas and products. The difference these days is that the details within the models being exchanged as objects within a mechatronic application become more advanced. What used to be just dimensions is now something that can be used within a complete simulation with programmable feedback and even application interfaces when a model includes application code. The interactive modeling is crucial to the design process, and it can occur in a mixed environment where real and virtual objects are combined. A real robotic arm may be coupled with a virtual assembly line, for example, if the current task is to determine if the hand on the robotic arm can reorient an object. The robotic arm might be involved in laser welding of end plates. The key is getting the virtual objects and their control counterparts to interact with the real objects with a code that is running on remote devices. The electromechanical control systems once designed for the factory floor have become ubiquitous. For example, a designer may answer a problem of vibration by adding a stiffener. In an integrated mechatronic process, however, that small mechanical change may increase the mass of the part; it also may affect how fast the control system ramps up motor speed and how long the part holds in place before returning.

Figure 8 shows how a design verifier can assess whether an object is used correctly within the system. In this instance,

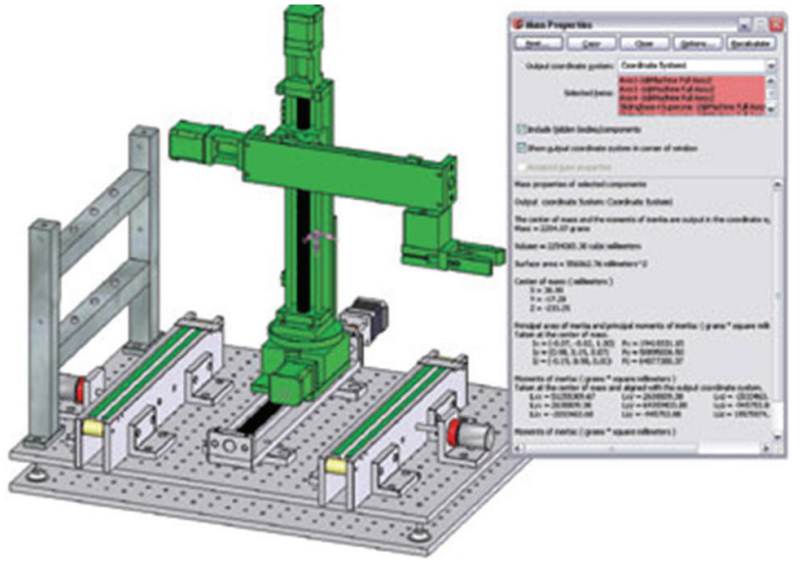

FIGURE 8: Simulation verification of torque load.

the simulation verified the correct torque load under variable loading conditions. IN the availability of design verifier, assertion blocks are able to be included within a model so the system can determine whether an object's use within a system is correct.

Many top mechatronic performers also use software that routes, tracks, and shares work. Most common are workflow tools, which automatically route work packages, warn about deadlines, and notify the right people of changes. Many companies make use of product data management tools to manage multidisciplinary bills of materials. Concurrent design is accomplished functionally using mechatronic approach. For example, engineers make assumptions about efficiency or how fast they can remove heat using certain construction techniques. Prototyping determines whether they hit their mark.

\subsection{Right for the First Time: Virtual Machine Prototyping.} The hardware in the loop facilitates the replacement of conventional mechanical motion control devices with digital devices. Mechanical systems are increasingly controlled by sophisticated electric motor drives that get their digital intelligence from software running on an embedded processor. Getting electromechanical designs right requires multidisciplinary teamwork and superb communication among team members. A decision like choosing the characteristics of a lead screw actuator has a ripple effect throughout the design and can impact the performance of other systems. To help facilitate a more integrated design process, we need to add motion simulation capabilities to CAD environments to create a more unified mechatronics workflow. Integrating motion simulation with CAD simplifies design because the simulation uses information that already exists in the CAD model, such as assembly mates, couplings, and material mass properties. Adding a high-level function block language for programming the motion profiles provides easier access to control those assemblies. This concept is known as "virtual machine prototyping" [31]. It brings together motion control software and simulation tools to create a virtual model of an electromechanical machine in operation. Virtual 
prototyping helps designers reduce risk by locating systemlevel problems, finding interdependencies, and evaluating performance tradeoffs. This is demonstrated in Figure 9 which shows the motion analysis for the green highlighted elements.

3.5. Evaluating Trade off. Simulations enable everyone to work on development before the first prototype is completed. Engineers can use force and torque data from simulations for stress and strain analysis to validate whether mechanical components are stiff enough to handle the load during operation. They also can validate the entire operating cycle for the machine by driving the simulation with control system logic and timing. They can calculate a realistic estimate for cycle time performance, which is typically the top performance indicator for a machine design and compare force and torque data with the realistic limitations of transmission components and motors. This information can help identify flaws and drive design iterations from within the CAD environment. Simulations also simplify evaluating engineering tradeoffs between different conceptual designs. For example, would a SCARA robot be preferable to the 4axis Cartesian gantry system? Simulations are faster and can be rerun whenever you make design changes. Consider an analysis of the torque load for the bottom lead screw actuator. If you violate the limits specified by the manufacturer, the mechanical transmission parts may not last for their rated life cycles. Using simulation software, you can find the mass of all the components mounted on the lead screw and determine the resulting center of mass by creating a reference coordinate system located at the center of the lead screw table and calculating the mass properties with respect to that coordinate system. With this information, you can calculate the static torque on the lead screw due to gravity caused by the overhanging load. Evaluating the dynamic torque induced by the motion is important because it tends to be much larger than the static torque load. Realistic motion profiles will help us to simulate inverse vehicle dynamics. This can provide more accurate torque and velocity requirements based on the motion profiles and the mass, friction, and gear ratio properties of the transmission.

\subsection{Mechatronically Designed Ambulatory Rehabilitation} Walker. The rehabilitation walker device designed and implemented by the authors in collaboration with a hospital is shown in Figure 10. This is an apparatus developed with the intent of aiding in the rehabilitation of hospital patients learning to walk again. This apparatus and control system are of industrial quality and would be reproducible in its entirety using off-the-shelf parts. The idea behind the rehabilitation walker is that it will relieve a certain percentage of body weight by carrying the patient in a harness which is attached to a hoist. The hoist is actively controlled using feedback from strain gauge sensors. As the patient walks around within the confines of the room-sized gantry, the hoist will follow the patient around. The overhead gantry is motorized in the $X$ and $Y$ directions. The closed loop motor control

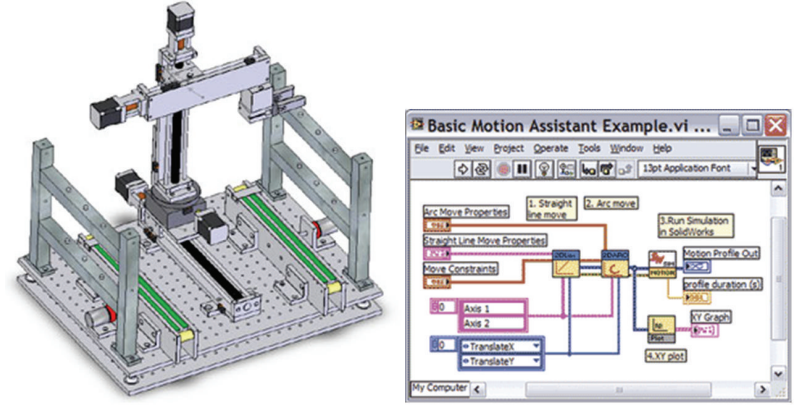

Figure 9: Evaluating "tradeoffs" in CAD environment [29].
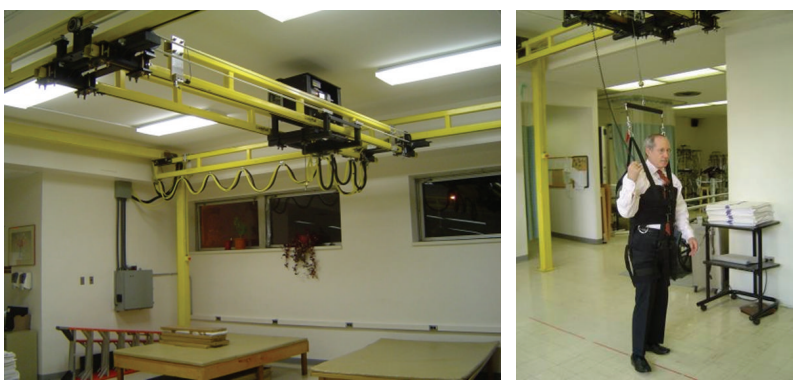

FIgURE 10: Mechatronic application for rehabilitation.

reacts to the feedback from multiaxis tilt sensors on the hoist line. If ever the patient were to fall, the hoist system would react and remove the full load of the patient's weight. The base of the control system consists of a National Instruments Compact RIO (reconfigurable input output) Programmable Automation Controller. The cRIO system is based on an FPGA backplane and a real-time controller. The backplane accepts modules which perform various I/O functions. The modules are chosen to interact with the rehabilitation walker sensors as well as handle the motor drive output signals. The motors are driven by industrial amplifiers, while position is tracked via quadrature encoder feedback. All programming of the system is done in LabVIEW (Figure 11).

3.7. Evidence-Based Diagnostics. In healthcare field, Internetbased systems are available to help doctors identify possible causes for patient symptoms. One such statistical diagnostic assistant, called "Isabel," was developed by a father who sought to change the diagnostic system that affected the way his daughter (Isabel) was treated. This system is basically an intuitive system that takes advantage of all previous diagnoses and provides the statistically most likely disease (fault) and treatment (repair). The application of the condition-based maintenance information system is available in army and military applications. The system has the ability to integrate information from on-board sensors and diagnostic equipment to develop fleet wide logistic and situational awareness, implementing condition-based maintenance service that will enhance the operation and effectiveness of the tactical and combat vehicles. 


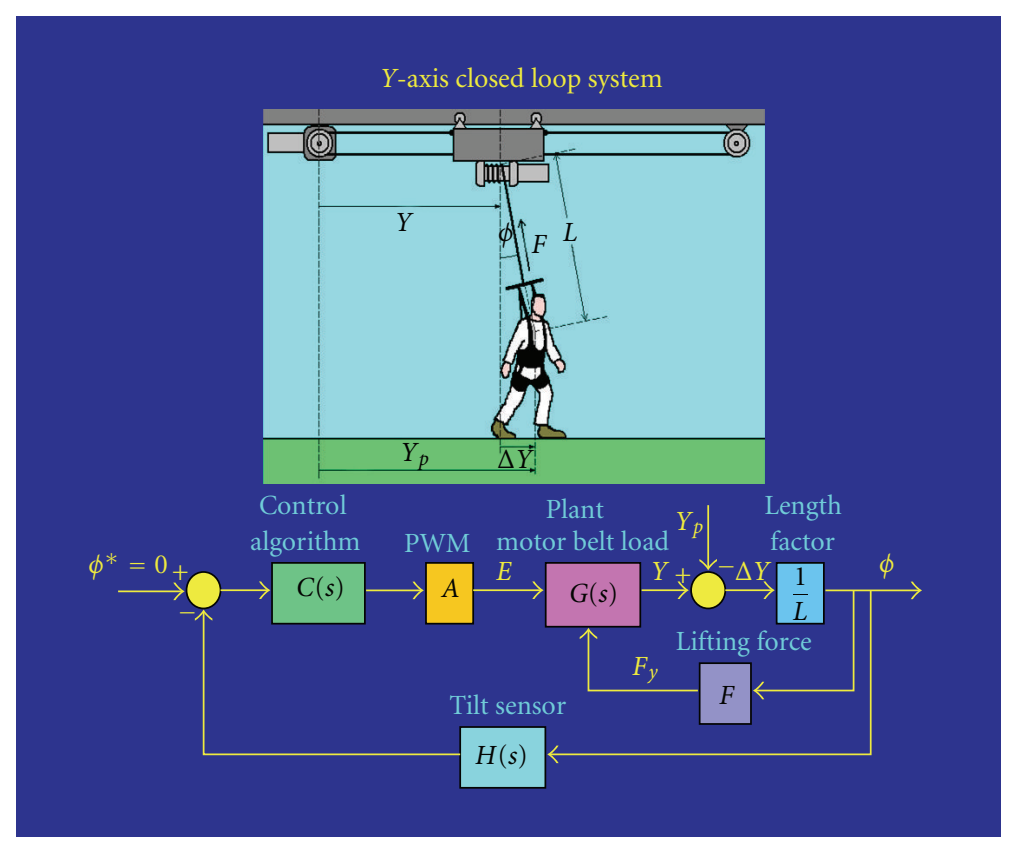

FIGURE 11: The $Y$-axis control loop system for the rehabilitation device "Navigator".

3.8. Smart Machining Platform. The defense and aerospace industries are facing the challenges of quality and manufacturing efficiency. The US Army has sponsored a program called SMPI, which is organized into three modules. The first module consists of development of smart test bed for the integration, validation, and demonstration of SMPI technology areas. The second module deals with extension of test bed to find synergy between two or more thrust areas. The third module deals with integration of various thrust areas into a complete smart machine capable of making the first part correctly.

3.9. E-Manufacturing. E-manufacturing is a system methodology that enables the manufacturing operations to successfully integrate with the functional objectives of an enterprise through the use of Internet, tether-free (i.e., wireless, web, etc.), and predictive technologies [32, 33]. E-manufacturing includes the ability to monitor the plant floor assets, predict the variation and performance loss to dynamically reschedule production and maintenance operations, and synchronize related and consequent actions to achieve a complete integration between manufacturing systems and upper-level enterprise applications. The Rockwell automation report outlines a statement of competencies that are required of world class companies. These are design, operate, maintain, and synchronize. E-manufacturing should include intelligent maintenance and performance assessment systems to provide reliability, dependability, and minimum downtime with equipment running smoothly at their highest performance.

\section{Conclusions}

Increasing demands on the productivity of machine tools and their growing technological complexity call for improved methods in future product development processes. This paper examines the application of integrated tools for setting up a virtual prototype at early phases of the development process. Starting with mechatronics design process, the paper looks at open architecture issues, interactive modeling, and virtual prototyping. Mechatronics is also influenced by intelligent devices for the online and realtime monitoring which includes diagnosis and control of processes. Recent advances of mechatronics in smart manufacturing and modifications and improvements to conventional designs by using a mechatronics approach are discussed.

Products will continue to move toward greater complexity and an increasing integration of mechanical and electronic functions, including the growing trend toward ubiquitous computing and embedded systems. The way in which companies design and manufacture products has evolved rapidly with the emergence of global supply chains. Increasingly, components and subsystems will be sourced from worldwide suppliers and will need to come together seamlessly in subassembly and final assembly operations. There will be many more challenges as manufacturing locations are added and eliminated to meet contract demands and hold down import duties on new global contracts. Also, suppliers and components will be increasingly rotated in and out of a design to meet the challenges of global supply chains and cost reduction pressures. Manufacturing will be far more fluid and will need to adjust rapidly to new locations, component suppliers, and design changes. For all of these reasons, advanced mechatronic tools will be required to assure high-quality manufacturing from the design for manufacture to the continuous monitoring and modification of the production processes. They will be essential for both high quality and profitability. 


\section{Conflict of Interests}

The authors have declared that no conflict of interests exists.

\section{References}

[1] Y. Li, J. Huang, and H. Tang, "A compliant parallel XY micromotion stage with complete kinematic decoupling," IEEE Transactions on Automation Science and Engineering, vol. 9, no. 3, pp. 538-553, 2012.

[2] Y. Yun and Y. Li, "Optimal design of a 3-PUPU parallel robot with compliant hinges for micromanipulation in a cubic workspace," Robotics and Computer-Integrated Manufacturing, vol. 27, no. 6, pp. 977-985, 2011.

[3] Y. Tang and Y. Li, "The software architecture of a reconfigurable real-time onboard control system for a small UAV helicopter," in Proceedings of the 8th International Conference on Ubiquitous Robots \& Ambient Intelligence (URAI '11), pp. 228-233, Songdo Convention, Incheon, Korea, November 2011.

[4] Y. Tang and Y. Li, "Development of a laboratory HILs testbed system for small UAV helicopters," in Proceedings of the IASTED International Conference on Robotics (Robo '11), pp. 428-436, Pittsburgh, Pa, USA, November 2011.

[5] J.-G. Wang and Y. Li, "Hybrid impedance control of a 3-DOF robotic arm used for rehabilitation treatment," in Proceedings of the 6th IEEE International Conference on Automation Science and Engineering (CASE '10), pp. 768-773, Ontario, Canada, August 2010.

[6] D. Shetty and R. Kolk, Mechatronic System Design, Thomson Engineering Publications, Toronto, Canada, 1998.

[7] G. Reinhart and M. Weissenberger, "Multibody simulation of machine tools as mechatronic systems for optimization of motion dynamics in the design process," in Proceedings of the IEEE/ASME International Conference on Advanced Intelligent Mechatronics (AIM '99), pp. 605-610, Atlanta, Ga, USA, September 1999.

[8] D. K. Miu, Mechatronics, Springer, Berlin, Germany, 1993.

[9] G. Reinhart, M. WeiBenberger, A. Sprenzel, J. Meinlschmidt, and P. Wagner, "Innovative entwicklung von werkzeugmaschinen -methoden und entwicklungswerkzeuge," in Synergy of Culture and Production, Vol. 1-Holistic Approach To Machine Tool Innovation, H. Y. Ito and E. F. Moritz, Eds., Artefact, Sottrum, Germany, 1997.

[10] K. Erkorkmaz and Y. Altintas, "High speed contouring control algorithm for CNC machine tools," in Proceedings of the ASME International Mechanical Engineering Congress and Exposition, pp. 463-469, November 1998.

[11] H. S. Cho and M. Y. Kim, "Optomechatronic technology: the characteristics and perspectives," IEEE Transactions on Industrial Electronics, vol. 52, no. 4, pp. 932-943, 2005.

[12] K. Kincade, "Atomic-force microscopy finds new role in the nano world," Laser Focus World, vol. 40, no. 4, pp. 87-91, 2004.

[13] N. Wakami, H. Nomura, and S. Araki, "Fuzzy logic for home appliances," in Fuzzy Logic and Neural Networks, C. H. Chen, Ed., pp. 21.1-21.23, McGraw- Hill, New York, NY, USA, 1996.

[14] T. J. Krupa, "Optical R\&D at the army research laboratory," Optics and Photonics News, vol. 11, no. 6, pp. 16-25, 2000.

[15] S. D. Robinson, "MEMS technology-micromachines enabling the all optical network," in Proceedings of the 51st Electronic Components and Technology Conference, pp. 423-428, Orlando, Fla, USA, June 2001.
[16] K. Tsuruta, Y. Mikuriya, and Y. Ishikawa, "Micro sensor developments in Japan," Sensor Review, vol. 19, no. 1, pp. 37-42, 1999.

[17] T. V. Higgins, "Optical storage lights the multimedia future," Laser Focus World, vol. 31, no. 9, p. 103, 1995.

[18] S. G. Anderson, "Smart cars take the high-tech road," Laser Focus World, vol. 32, no. 6, p. 117, 1996.

[19] T. G. McDonald and L. A. Yoder, "Digital micromirror devices make projection displays," Laser Focus World, vol. 33, no. 8, pp. S5-S8, 1997.

[20] H. S. Cho, Opto-Mechatronic Systems Handbook: Techniques and Applications, CRC Press, Boca Raton, Fla, USA, 2003.

[21] D. A. Bradley, D. Dawson, N. C. Burd, and A. J. Loader, Mechatronics: Electronics in Products and Processes, Chapman \& Hall, London, UK, 1991.

[22] Y. Altintas, C. Brecher, M. Week, and S. Witt, "Virtual machine tool," CIRP Annals, vol. 54, no. 2, pp. 651-674, 2005.

[23] H. Van Brussel, P. Sas, I. Németh, P. De Fonseca, and P. Van Den Braembussche, "Towards a mechatronic compiler," IEEE/ASME Transactions on Mechatronics, vol. 6, no. 1, pp. 90105, 2001.

[24] M. F. Zaeh, T. Oertli, and J. Milberg, "Finite element modelling of ball screw feed drive systems," CIRP Annals, vol. 53, no. 1, pp. 289-293, 2004.

[25] G. Pritschow, "On the influence of the velocity gain factor on the path deviation," CIRP Annals, vol. 45, no. 1, pp. 367-371, 1996.

[26] Y. Altintas and M. Weck, "Chatter stability of metal cutting and grinding," CIRP Annals, vol. 53, no. 2, pp. 619-642, 2004.

[27] R. Gurbuz, "Mechatronics approach for desk-top CNC milling machine design," Diffusion and Defect Data B, vol. 144, pp. 175-180, 2009.

[28] Siemens PLM Software, CHICAGO (IMTS), 2010, http://www .siemens.com/industryautomation.

[29] B. M. Cleery and N. Mathur, "Right the first time," Mechanical Engineering, vol. 130, no. 6, pp. 30-34, 2008.

[30] W. Wong, Muticore Matters with Mechatronic Models Electronic Design, 2008.

[31] NI LabVIEW-Solid Works mechatronics toolkit, http://www .ni.com/mechatronics/.

[32] A. Ali, Z. Chen, and J. Lee, "Web-enabled platform for distributed and dynamic decision-making systems," International Journal of Advanced Manufacturing Technology, vol. 38, no. 1112 , pp. 1260-1270, 2008.

[33] J. Lee, "E-manufacturing-fundamental, tools, and transformation," Robotics and Computer-Integrated Manufacturing, vol. 19, no. 6, pp. 501-507, 2003. 

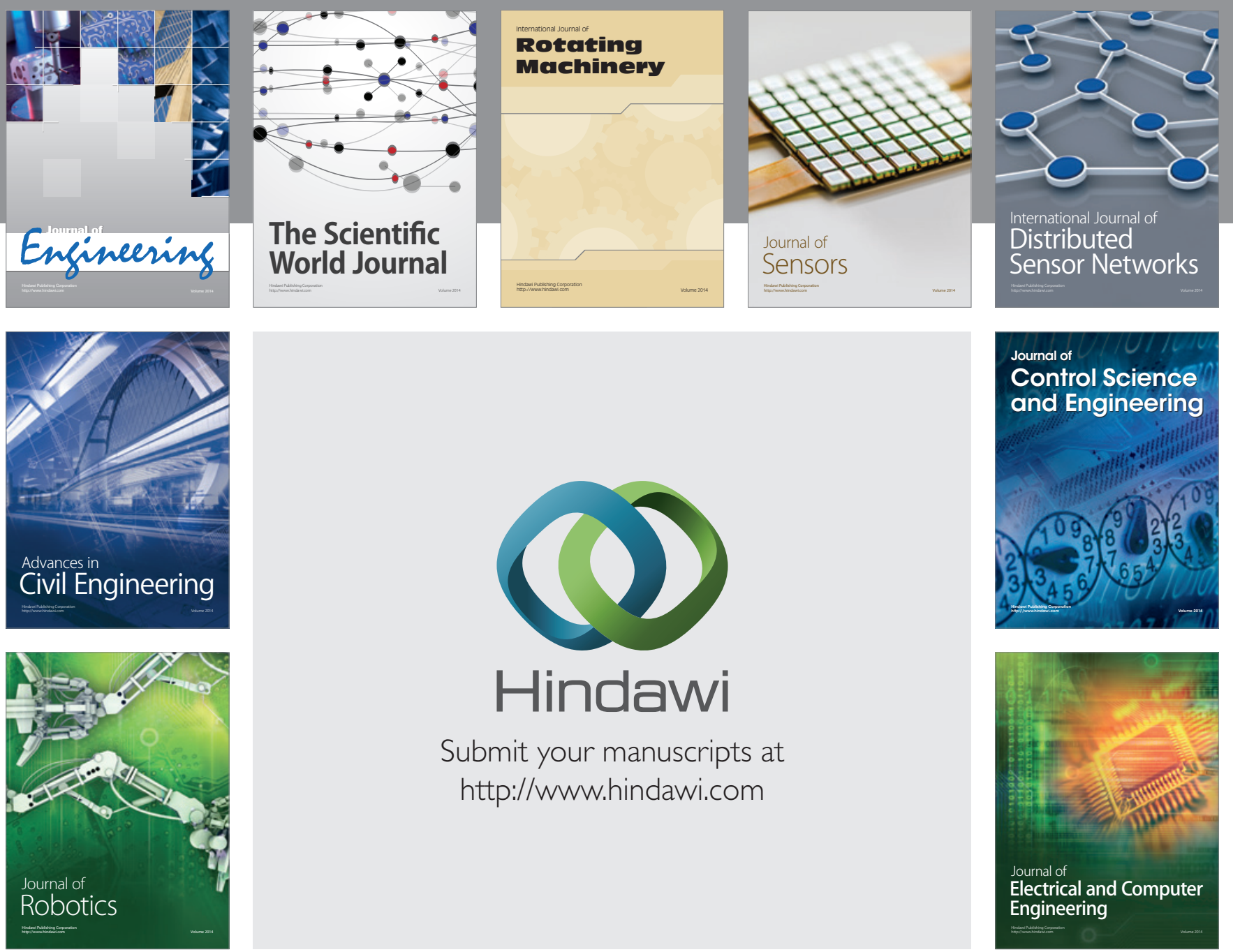

Submit your manuscripts at

http://www.hindawi.com
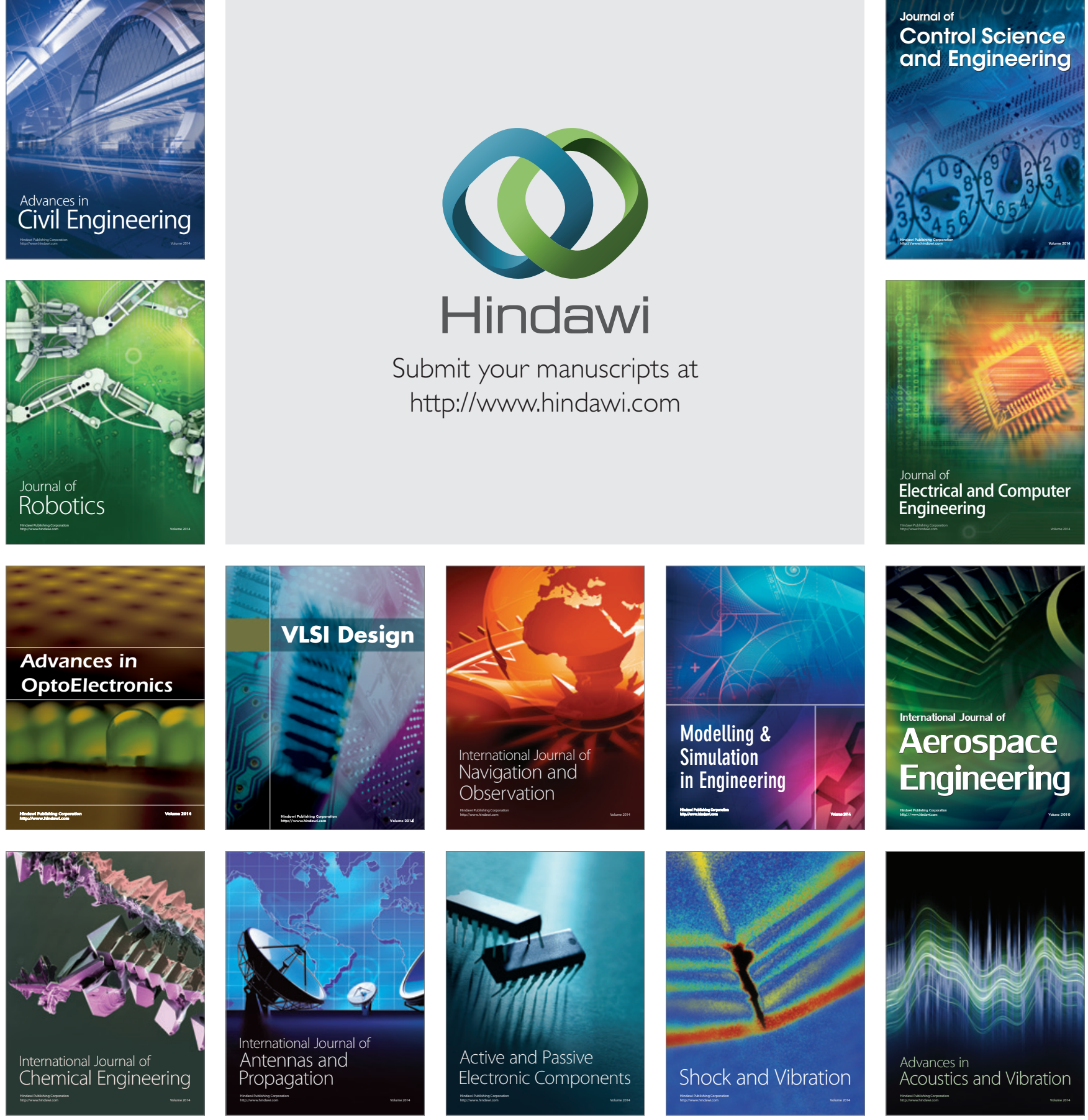